Voix et Images

voixetimages

\title{
La Fleur aux dents : Un vrai film pour la télévision
}

\section{Gilles Thérien}

Volume 1, numéro 2, décembre 1975

\section{Raoul Duguay}

URI : https://id.erudit.org/iderudit/290084ar

DOI : https://doi.org/10.7202/290084ar

Aller au sommaire du numéro

\section{Éditeur(s)}

Les Presses de l'Université du Québec

ISSN

0318-9201 (imprimé)

1705-933X (numérique)

Découvrir la revue

Citer ce compte rendu

Thérien, G. (1975). Compte rendu de [La Fleur aux dents : Un vrai film pour la télévision]. Voix et Images, 1(2), 295-301. https://doi.org/10.7202/290084ar d'utilisation que vous pouvez consulter en ligne.

https://apropos.erudit.org/fr/usagers/politique-dutilisation/ 


\section{La Fleur aux dents}

\section{Un vrai film pour la télévision}

La Fleur aux dents offre deux axes de réflexion. II s'agit d'abord d'un film fait pour la télévision et quı se veut tel. C'est aussi un roman de Gilles

3. Je sais que ma description est quelque peu caricaturale. La problématique est plus complexe que je veux bien le laisser croire et la position des différents groupes n'est pas aussi tranchée que je l'affirme. A preuve les discussions de Chroniques sur la langue et plus récemment les déchirements au niveau de l'ANEQ concernant les priorités de leur action contestataire (Bill 22 ou hausse des tarifs dans le métro et les autobus de Montréal ?). 
Archambault ${ }^{1}$ et, à ce titre, il nous intéresse dans la mesure où le «texte" du film ${ }^{2}$ et celui du roman ont des points communs et d'autres divergents.

\section{LE FILM}

Quand cela est possible, il faut faire connaître la fiche technique du film. Il est alors évident que certaines données délimiteront les résultats à un niveau ou à un autre. L'entière disponibilité de Thomas Vamos nous a permis d'élaborer une fiche technique qui servira de toile de fond à l'analyse.

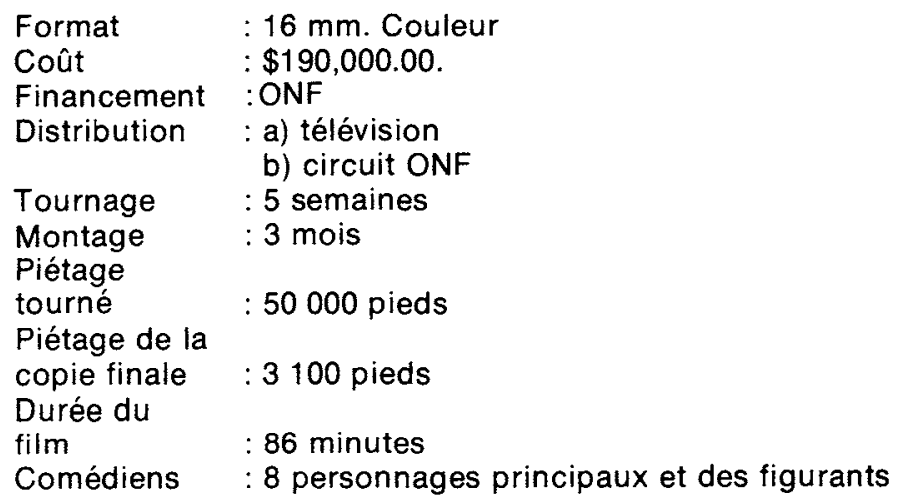

Comme on peut le constater, il s'agit d'un budget modeste... si l'on songe que le budget moyen actuel est plutôt de $\$ 350000.00$. Pourtant le film n'a pas été fait dans des conditions difficiles puisque le piétage tourné est très élevé, une proportion d'environ quinze pour un et que la distribution est importante. Ajoutons que le film dispose de tous les moyens techniques essentiels et que les délais - tournage, montage - sont normaux. II n'y aura donc pas lieu, dans ce cas-ci, d'imputer à "la machine " les défauts du produit.

La Fleur aux dents est fait pour la télévision, d'où son format $16 \mathrm{~mm}$ plutôt que 35. Mais cela est loin d'être la seule conséquence. Le film a une durée (86 minutes) compatible avec la grille horaire de la télévision. La longueur d'un film est un problème très délicat. De la part de l'auteur, il pourrait se résumer au temps nécessaire à la narration... Les uns sont plus lents que d'autres; certaines narrations sont plus complexes. Sous l'angle du mode de diffusion, le problème devient fort différent. Si le cinéma a tendance à allonger la durée du spectacle, deux heures contre une heure trente, la télévision oblige, elle, à une durée précise qu'on peut incorporer

1. La Fleur aux dents, Montréal, Le Cercle du livre de France, 1971.

2. Réalisé par Thomas Vamos. 
sans trop de difficultés aux grilles de programme. C'est donc avec une durée précise en tête que le film, c'est-à-dire la dramaturgie, a été conçu.

Ceci ne suffirait toutefois pas à faire de ce film un film de télévision. Certaines expériences récentes l'ont montré. Le visionnement à la télévision de films spectaculaires ou d'ambiance, tels le Parrain, Cabaret, État de siège, pour n'en nommer que quelques-uns, pose bien le problème de la différence entre le petit et le grand écran. Le spectateur du petit écran domine le spectacle. Ce dernier a alors un cadre tout à fait familier, très loin de la magie du cinéma où c'est l'écran qui domine le spectateur. Les séries américaines ont bien compris cette différence et évitent à la télévision tout ce qui, dans un spectacle, a un caractère impressionniste. La télévision est un médium pour l'archi-vrai, information, témoignages, reportages ou l'archi-faux, les variétés, les jeux, etc. La série, le film pour la télévision, doivent se conformer à ces catégories. L'effet de réel télévisuel en dépend.

C'est dans le même esprit que sera modifiée l'écriture cinématographique proprement dite. Les plans doivent être assez rapprochés, aisément intimistes, les séquences ne peuvent être ni trop longues, ni trop courtes étant donné qu'à la télévision, la concentration est difficile, distendue. II est hors de question d'utiliser les procédés classiques de fondus, de montages en parallèle, etc., puisque le spectateur y perdra de vue la trame narrative. En outre, il ne faut jamais oublier qu'au moment prévu par la station, et non par le cinéaste, on insérera des messages publicitaires plus ou moins compatibles avec le film ou avec les niveaux de l'émotion véhiculée.

Si le film trop cinématographique passe mal à la télévision, l'inverse se vérifie aussi. Le film de télévision ne doit pas nécessairement être présenté au cinéma. Ses gros plans deviendront gênants, son montage un peu trop simpliste: le risque est grand que ce qui assurait un succès à la télévision devienne la cause même de l'échec au cinéma. Ces réflexions tendent à montrer qu'il existe deux orientations différentes pour un même produit selon qu'il utilise un type particulier de diffusion, la télévision ou la salle de cinéma. L'expérience de Vamos consiste principalement à avoir tenté de respecter ce choix.

"L'Inévitable échec de Georges Lamontagne", tel pourrait être le sous-titre du film. Principalement centré sur ce personnage, père de famille de quarante ans, technicien de la radio à CKMM, le récit ne comporte pas que cette trame. On retrouve aussi le récit des problèmes personnels de Louise, sa femme, l'histoire de la grossesse et du mariage de sa fille Marie-France. La station CKMM et son super animateur (séducteur-conet-névrosé) fournit une autre trame. La poursuite du rêve de Georges Lamontagne de devenir journaliste et finalement l'échec qui s'ensuivra constituent des noyaux narratifs importants. L'apparente simplicité de la trame linéaire du film est un leurre. Quand le film se termine, un certain nombre de conclusions sont possibles: elles concernent toutes Georges Lamontagne. Qu'en est-il du reste? Le film laisse le spectateur sur sa faim. 
Ces multiples trames qui vont ou ne vont pas vers une résolution constituent en fait l'essence même de la dramaturgie. En général, on oppose au film dit linéaire, un film à montage parallèle. Or, l'essentiel de la dramarturgie en tant que structure consiste justement à inscrire dans le récit principal de multiples récits secondaires dont la principale fonction est de permettre l'évolution du personnage autrement que "par ses propres forces". Les facettes de Georges Lamontagne sont explorées sans que la trame générale ne disparaisse mais elle est constamment modifiée. La linéarité devient toute relative. Un des récits secondaires, celui de la présence du mort Alain Turcotte.dans la mémoire de Georges et de Louise Lamontagne, expose assez bien son intégration au récit principal: Georges et Louise «aiment " Turcotte, le brillant journaliste décédé trop jeune. Toutefois cette intégration est d'autant plus visible qu'elle s'accompagne d'une ambiguïté importante sur le rôle de ce Turcotte dans la vie du couple. Turcotte était-il l'amant de Louise? Réponse de Vamos: non. Ce récit secondaire est à la fois dans et hors de la trame principale... et c'est ce genre de défaut de tissage qui nous permet d'apprécier l'emboîtement des récits.

Claude Jutra joue le personnage de Lamontagne. II en fait un être introverti, plus capable d'humour que de chaleur; Louise, sa femme, est chaleureuse mais aussi traumatisée par le vieillissement. Marie-France et son ami Michel sont comme des "ovnis", un peu caricaturaux. Liliane et Sylvio sont rafraîchissants par leur vérité... Quant aux autres, c'est-à-dire l'équipe de CKMM, ils tiennent de quelque faune monstrueuse où l'intelligence n'a pas été accordée en prime. Tous ces personnages parlent, bavardent; ils poursuivent, chacun, une logique différente, et s'intègrent à ce monde brisé qui évolue plus ou moins malgré lui.

Comme tout bon film, la Fleur aux dents substitue au mot «FIN", une image de fin, long plan d'hélicoptère qui rapetisse Georges Lamontagne à la dimension d'un insecte. Cette image est un peu lourde, trop kafkaïenne pour un film qui est, somme toute, léger, marqué par un humour grinçant mais qui ne contient pas de brisures tragiques. Le mythe de la clôture du texte par un mot ou par une image demeure très fort. Il est encore que très peu permis de terminer sur trois points de suspension.

\section{DU ROMAN AU FILM}

La Fleur aux dents est d'abord un roman de Gilles Archambault qui date de 1971. Sa facture est relativement simple. En alternance sont présentés des monologues intérieurs de Georges Lamontagne et une sorte de récit, plus bref, plus objectif, qui vient définir, décrire le monde de Georges Lamontagne.

Audouard, scénariste français réputé, disait qu'il ne fallait jamais adapter un classique à l'écran. Le public s'est déjà fait une idée des per- 
sonnages d'un grand roman et le danger du film est de ne pas correspondre à cette idée. À part quelques moments de grâce, il en est toujours ainsi. Le roman dont la puissance évocatrice est moindre fait par ailleurs un excellent document de base pour un scénario. Autre difficulté, le problème du style. Certains romans valent par leur style, par leur écriture. Si le cinéaste ne trouve pas de transposition équivalente, le résultat sera désastreux. Qu'on pense à l'Ulysse de Joyce et à la navrante version de Joseph Strick. Par ailleurs, Toby Dammit de Fellini restitue l'atmosphère de l'œuvre entière d'Edgar Allan Poe, sans se plier à la lettre au récit d'une nouvelle particulière.

Ici, le problème de l'adaptation est plus réduit. L'auteur ne s'est pas mêlé de la scénarisation de son roman. II n'a donc pas eu à faire lui-même l'inévitable choix entre tel ou tel élément. Toutefois, sur le plan du style, un élément pouvait recevoir plusieurs traitements: il s'agit du long monologue de Georges Lamontagne, monologue qui semble meubler les moments vides laissés par l'utilisation du studio. Sur le plan de l'écriture romanesque, il s'agit d'un récit intérieur; au cinéma, ce monologue aurait pu devenir une sorte de commentaire "off", venant contredire l'action ici ou là, lui offrant une sorte de contrepoint. Cette solution n'a pas été retenue. On lui a préféré une solution plus bavarde, du moins en apparence, mais qui, dans son bavardage même - les blagues, les discussions inutiles... rejoint le même sentiment que celui que vise Archambault, l'ennui.

Le roman s'intéresse à la vie de Georges Lamontagne, petit technicien de studio, dont le grand rêve est de ressembler à son ami Alain, "flambeur» par excellence, qui se suicide parce qu'il en a assez. Georges se donne pour un libéral, il essaie de réussir en se tenant aux idées d'Alain et en voulant devenir, lui aussi, un grand reporter. Il échoue, se fait "vider» de la station de radio, ce qui lui permet, prétend-il, de réaliser enfin son rêve: devenir épicier.

\section{LES SUBSTITUTIONS}

La très grande liberté d'adaptation prise par Vamos et son coscénariste Pierre Turgeon a engendré un certain nombre de substitutions fort intéressantes. Sur le plan même de l'écriture, notons simplement que le film s'ouvre avec Georges qui sort de la toilette et boutonne sa braguette. La première phrase du roman est: «Dans la vie, je me débrouille assez bien." Bien inconsciemment, l'iconique confère un milieu concret à la vie dans laquelle on se débrouille. Et tout au long du film, les lieux, les objets viendront banaliser le quotidien: vieille voiture, costume traditionnel, quartier "classe moyenne", etc.

La substitution la plus importante demeure celle du personnage principal, Georges Lamontagne. Dans le roman, il s'agit d'un être extraverti, ayant des aventures à gauche et à droite, reconnaissant à sa femme 
et à sa fille leur liberté sexuelle. Il est assez bien intégré au monde de la radio. Lamontagne-Jutra est un introverti, timoré, sans grande imagination et visiblement pas le maître chez lui. L'interprétation a été voulue ainsi par Vamos et si ce choix s'explique, ce n'est pas par le roman mais plutôt par le film lui-même. Ce qui, en effet, n'était qu'un hobby plus ou moins secondaire dans le roman devient une trame importante du film. Lamontagne veut faire une série d'émissions sur la "révolution tranquille». Il y consacre tout son temps libre et c'est l'échec de ce travail qui l'amènera à se recycler du monde des idées au monde de la matière en devenant une sorte de contremaître-électricien chargé d'inspecter les relais de la station. L'échec de Georges Lamontagne, c'est aussi celui de la révolution tranquille... son recyclage est celui de plusieurs personnes qui, durant ces années, ont cru (tout simplement!). L'inspecteur des relais est parent avec le réformiste Godbout, quelques sous-ministres devenus fédéralistes ou retournés à la pratique du droit. Peu d'épiciers toutefois!

Dans le roman, la femme de Lamontagne a aussi ses amants et un grain de culpabilité. Dans le film, elle n'a gardé que la culpabilité et au lieu de s'adonner à l'amour, elle pratique le transfert sur la personne d'un psychiatre. Ce dernier se trouvait dans le roman en meilleure posture, soit celle de conseiller-des-ondes s'adressant à tous et chacun. Louise Lamontagne semble plus être la proie du vieillissement qu'en plein épanouissement, et ses explosions féministes sont comme des symptômes du retour d'âge, sauf quand le scénariste lui fait lancer maladroitement l'injure "phallocrate".

Mentionnons enfin que l'amant-futur-époux de la fille de Georges est l'ancien amant de la maîtresse d'un soir de Georges. Le cinéma réduit le jeune à son cynisme et la maîtresse se voit parée d'une sorte d'auréole.

$\mathrm{Si}$, dans le roman, la triade, Georges Lamontagne - Louise, sa femme - et leur fille Marie-France, manifestait une sorte de liberté et s'installait dans une sorte d'équilibre, le film nous ramène, avec plus d'humour, il est vrai, à un schéma québécois bien connu. Louise est la femme abandonnée et confessée (par le psychiatre) mais qui possède des secrets (ceux de Marie-France). Georges est l'homme dominé à la maison et au travail et ce n'est que dans ses activités parallèles ou dans l'humour qu'il parvient à marquer son opposition. La fille se range du côté de la mère de même que la maîtresse d'un soir. II ne reste à Georges que les tentations, c'est-à-dire, la petite secrétaire du patron. Domination des femmes, échec de l'homme et de la révolution. Heureusement qu'on en rit un peu!

\section{CONCLUSION}

Le film se présente donc comme un film de télévision et, à ce titre, il sert au téléspectateur québécois une image de lui-même. Dans sa facture, son désir de dénoter sur le plan iconique un monde référentiel connu, il 
permet vraisemblablement à une partie de la population de se reconnaître, un peu comme les films sur les femmes ont atteint aussi cet objectif. (Bien sûr, le spectateur pourra toujours sortir de chez lui et aller assister au cinéma à des spectacles qui l'extraient du quotidien.) A la limite, la télévision est peut-être le lieu privilégié du cinéma "réaliste" québécois; aussi, l'expérience, bien consciente chez Vamos, est à suivre.

Le traitement d'un roman au cinéma nous permet ainsi d'aborder une grille d'analyse qui tienne compte des deux média et de ce qui, dans le cinéma, appartient à l'univers romanesque. On peut dès lors distinguer trois niveaux: l'iconique, que l'on ne retrouve qu'au cinéma, l'indiciel qui marque la narration de différentes façons selon qu'elle s'appuie sur l'iconique ou sur un discours (dans le roman) et le symbolique qui engage, de part et d'autre, les procédés rhétoriques et stylistiques propres aux deux modes d'expression.

C'est dans le domaine du savoir d'abord que la comparaison de textes différents par leur écriture (roman-cinéma) puise sa justification... mais le savoir engendre le pouvoir et c'est la réflexion sur les écritures spécifiques qui en profitera.

Gilles Thérien 